\title{
An Electromechanical Pendulum Robot Arm in Action: Dynamics and Control
}

\author{
A. Notué Kadjie, P. R. Nwagoum Tuwa, and Paul Woafo \\ Laboratory of Modelling and Simulation in Engineering, Biomimetics and Prototypes, Department of Physics, \\ Faculty of Science, University of Yaoundé I, P.O. Box 812, Yaoundé, Cameroon
}

Correspondence should be addressed to Paul Woafo; pwoafol@yahoo.fr

Received 22 June 2017; Revised 18 October 2017; Accepted 8 November 2017; Published 5 December 2017

Academic Editor: Francesco Pellicano

Copyright (C) 2017 A. Notué Kadjie et al. This is an open access article distributed under the Creative Commons Attribution License, which permits unrestricted use, distribution, and reproduction in any medium, provided the original work is properly cited.

\begin{abstract}
The authors numerically investigate the dynamics and control of an electromechanical robot arm consisting of a pendulum coupled to an electrical circuit via an electromagnetic mechanism. The analysis of the dynamical behavior of the electromechanical device powered by a sinusoidal power source is carried out when the effects of the loads on the arm are neglected. It is found that the device exhibits period-n T oscillations and high amplitude oscillations when the electric current is at its smallest value. The specific case which considers the effects of the impulsive contact force caused by an external load mass pushed by the arm is also studied. It is found that the amplitude of the impulse force generates several behaviors such as jump of amplitude and distortions of the mechanical vibration and electrical signal. For more efficient functioning of the device, both piezoelectric and adaptive backstepping controls are applied on the system. It is found that the control strategies are able to mitigate the signal distortion and restore the dynamical behavior to its normal state or reduce the effects of perturbations such as a short time variation of one component or when the robot system is subject to noises.
\end{abstract}

\section{Introduction}

Pendulum motion-driven systems have been intensively studied recently by both industries and research institutes because of their applications in different fields [1-10]. Some of these studies concern the analysis of the dynamical states and the development of control strategies to stabilize the dynamical state to a prescribed state. These pendulum models comprise the downward pendulum [5], horizontal pendulum [6], inverted pendulum [7], spherical pendulum $[8]$, the flexible pendulum $[9,10]$, the pendulum excited by an RLC circuit based on nonlinear shaker [11], and rotating pendulum [12]. When the pendulum is coupled to an electrical part (electromechanical pendulum), its applications with and without control are more interesting in robotics and other fields of engineering. This is due to some particular dynamical states (periodic, quasiperiodic, and chaotic states) that the electromechanical pendulum can generate because of intrinsic angular nonlinearity or due to natural or imposed nonlinearities in the electrical part [5, 9-12].
The working state of a system with particular dynamics can be modified because of the interaction with its environment or the application of some constraints or control laws. In this line, recent years have seen the development of various control strategies applied on electrical, mechanical, electromechanical, and even biological systems: some examples are the adaptive control [13], active control [14], the classical and active-backstepping controls $[15,16]$, and the sliding mode control [17]. An interesting contribution dealing with chaos control of a double pendulum arm powered through an RLC circuit is reported in [11] where the authors used the state-dependent Riccati equation control and the nonlinear saturation control techniques to suppress chaos in the dynamics of the double pendulum arm. Due to its importance for engineering and robotic applications, the control of pendulum motion has been intensively studied using various approaches, including passivity-based control [18], nonlinear control [19, 20], sliding mode control [21], motion control of two pendulums [22], and bifurcation control [23].

In this work, the dynamics and the control of an electromechanical pendulum with rigid and constant length 


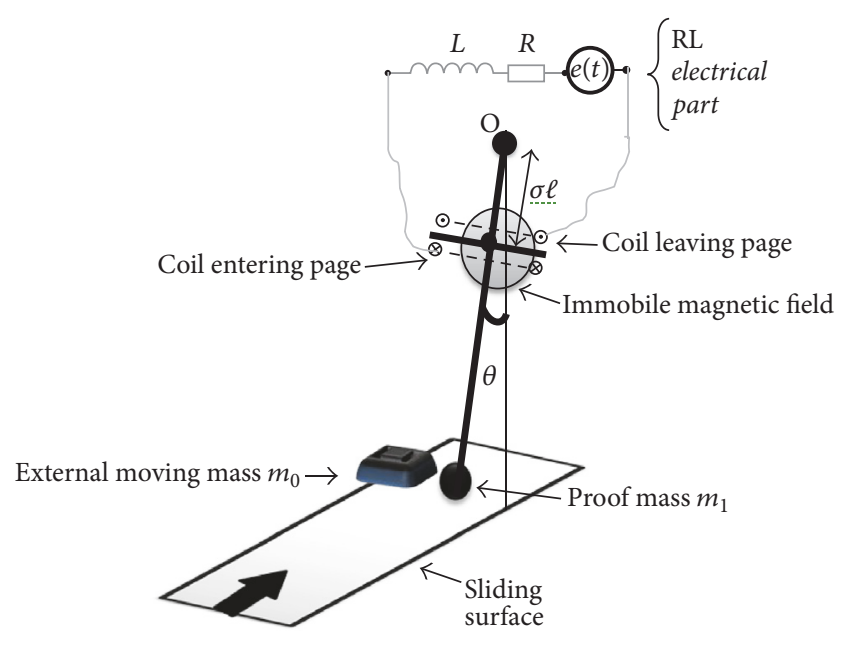

FIGURE 1: Schematic representation of the electromechanical system.

are studied. The pendulum is coupled to an electrical part through an electromagnetic link. The dynamics considers the effect of a periodic impulsive force due to the instantaneous shock between the pendulum arm and external load masses arriving periodically. This is described mathematically by a pulse-like excitation added to the initial sinusoidal electrical excitation. In terms of the load mass, the critical electrical signal amplitude leading to the displacement of the mass is evaluated. In view of optimizing the action of the pendulum arm by counterbalancing the collision effects due to the arriving loads, a pulse-like activation signal acts periodically on the pendulum arm. Finally, an adaptive backstepping method, based on the automatic variation of an intrinsic parameter, has been developed either to control the perturbations caused by the collision of the pendulum with the load masses or to counterbalance the disturbances generated by unwanted temporal variations of some parameters of the robot device.

The work is structured as follows. Section 2 describes the electromechanical pendulum robot arm and analyzes the dynamics of the arm when the action of the load is neglected. Section 3 considers the situation where the effect of the periodic actions of the load is taken into account. In order to optimize the working conditions of the robot arm, a control strategy consisting of sending pulse-like signals following the detection of the load arrival is also considered in this section. Because the device can be subject to an unknown time variation (of regular or stochastic nature) of some of its parameters, a backstepping adaptive method is used in Section 4 to reduce the effects of these perturbations on the system. Finally, Section 5 concludes the work.

\section{Description, Modelling, and Dynamics of the Electromechanical Pendulum with Negligible Interaction with the Moving Mass}

Figure 1 shows the electromechanical robot arm. It is constituted of a pendulum with a rod of length $\ell$ and the spherical proof mass $\left(m_{1}, r_{1}\right)$, coupled electromagnetically to an electrical circuit. The electrical part of the system is constituted of a coil wired around an iron core rigidly fixed on the pendulum over a length $\sigma \ell$ of the pendulum whose rotation axis passes at the point $O . \sigma$ is a constant indicating the proportion of the coiled rod length inside the magnetic field.

The ends of the coil are connected, through a LR circuit, to an electrical source able to deliver different types of signals. Moreover, the coil is immersed in a constant and permanent magnetic field delivered by two identical and immobile magnets. When the electrical source is activated, the rod moves with the coil, because of Laplace's force, in parallel to the magnets surface and at an equidistant plane between the magnets. Consequently, the pendulum motion can be used to give the motion or to change the direction of an external load mass $m_{0}$ which is positioned periodically at the equilibrium state of the pendulum (see Figure 1). Giving or changing the motion of an external mass results from the collision between the masses $m_{0}$ and $m_{1}$. The parameters values (and their units) of the device are listed in Table 1.

The sinusoidal form of the electrical signal $e(t)$ is considered and the effect of the interaction with the moving mass $m_{0}$ is neglected. The mathematical investigation and the numerical simulation are used to analyze the dynamical behaviors of the device. Bifurcation diagrams and Lyapunov exponent are plotted to show the different dynamical states of the system when the excitation amplitude changes.

According to Newton's second law and Kirchhoff's law, the electromechanical equations with sinusoidal excitation are given as [5]

$$
\begin{gathered}
L \frac{d i}{d t}+R i+\frac{n B b^{2}}{2} \frac{d \theta}{d t}=e_{0} \cos (\Omega t) \\
J \frac{d^{2} \theta}{d t^{2}}+\omega_{1}^{2} \sin \theta+\mu \frac{d \theta}{d t}=\frac{n B b^{2}}{2} i,
\end{gathered}
$$

where $J=m_{1}\left((2 / 5) r^{2}+\ell^{2}\right)+(1 / 3) m_{2} \ell^{2}+(\sigma \ell)^{2} m_{3}$ is the total inertia moment of the system and $\omega_{1}^{2}=\left(m_{1}+m_{2} / 2+\right.$ $\left.\sigma m_{3}\right) g \ell$ and $L=\left(\mu_{0} \mu_{r} n^{2} / \pi\right) \sqrt{a^{2}+b^{2}}$ [24]. $i$ is the electrical current, $\theta$ is the angle swept by the pendulum relative to the vertical reference position. $e_{0}$ and $\Omega$ are the amplitude and the frequency of the electrical excitation. $d / d t$ is the derivative with respect to time.

Let us use the following notations and relations:

$$
\begin{aligned}
& (\cdot)=\frac{d}{d \tau} ; \\
& \left(\text { ) }=\frac{d^{2}}{d \tau^{2}}\right. \\
& y=\frac{\theta}{\theta_{0}} \\
& x=\frac{i}{i_{0}}
\end{aligned}
$$


TABLE 1: Physical parameters of the electromechanical system.

\begin{tabular}{|c|c|c|c|}
\hline & Parameter & Value & Units \\
\hline \multirow{2}{*}{ Spherical proof mass } & Mass: $m_{1}$ & 0.5 & $\mathrm{~kg}$ \\
\hline & Radius: $r_{1}$ & 0.01 & $\mathrm{~m}$ \\
\hline \multirow{7}{*}{ Rectangular coil } & Thickness: $a$ & 0.05 & $\mathrm{~m}$ \\
\hline & Height: $b$ & 0.2 & $\mathrm{~m}$ \\
\hline & Resistance: $R$ & 20 & $\Omega$ \\
\hline & Number of coil turns: $n$ & 500 & 1 \\
\hline & Magnetic field intensity: $B$ & 1 & $\mathrm{~T}$ \\
\hline & Proportion of the coiled rod: $\sigma$ & 0.2 & 1 \\
\hline & Coil mass: $m_{3}$ & 0.1 & $\mathrm{~kg}$ \\
\hline \multirow{3}{*}{ Rod } & Viscous friction coefficient: $\mu$ & 0.1 & $\mathrm{~N} \mathrm{~s} / \mathrm{m}$ \\
\hline & Mass: $m_{2}$ & 0.4 & $\mathrm{~kg}$ \\
\hline & Length: $\ell$ & 0.5 & $\mathrm{~m}$ \\
\hline
\end{tabular}

$$
\begin{aligned}
\omega_{0}^{2} & =\frac{\omega_{1}^{2}}{J \theta_{0}} ; \\
\lambda & =\frac{R}{L \omega_{0}} ; \\
\gamma_{e} & =\frac{n B b^{2} \theta_{0}}{2 L i_{0}} ; \\
\gamma_{m} & =\frac{n B b^{2} i_{0}}{2 J \omega_{0}^{2} \theta_{0}} ; \\
E_{0} & =\frac{e_{0}}{L \omega_{0} i_{0}} ; \\
\omega & =\frac{\Omega}{\omega_{0}} ; \\
\tau & =\omega_{0} t ; \\
\beta & =\frac{\mu}{J \omega_{0}} .
\end{aligned}
$$

$\omega_{0}, \theta_{0}$, and $i_{0}$ are, respectively, the reference frequency, the reference angle, and reference current.

Equations (1) take the following dimensionless form:

$$
\begin{aligned}
\dot{x}+\lambda x+\gamma_{e} \dot{y} & =E_{0} \cos (\omega \tau) \\
\ddot{y}+\beta \dot{y}+\sin \left(\theta_{0} y\right) & =\gamma_{m} x .
\end{aligned}
$$

With the values of the parameters given in Table 1 , one obtains the following numerical values: $\lambda=2.67, \gamma_{e}=1.905, \beta=$ 0.204 , and $\gamma_{m}=0.895$.

In (4), the angular nonlinearity appears with the presence of the trigonometric function. This term complicates the analytical investigation. Consequently, only the case of small and average amplitude of vibration is considered for the mathematical investigation. The following approximation can thus be used (from the Taylor expansion formula):

$$
\sin \left(\theta_{0} y\right) \approx \theta_{0} y-\frac{1}{6}\left(\theta_{0} y\right)^{3}
$$

Equations (3) and (4) can thus be written as

$$
\begin{aligned}
\dot{x}+\lambda x+\gamma_{e} \dot{y} & =E_{0} \cos (\omega \tau) \\
\ddot{y}+\beta \dot{y}+\alpha_{1} y+\alpha_{2} y^{3} & =\gamma_{m} x
\end{aligned}
$$

with $\alpha_{1}=\theta_{0}$ and $\alpha_{2}=-(1 / 6) \theta_{0}^{3}$.

The harmonic balance method is used to solve (6) to find the sinusoidal approximations of the angular displacement and electrical current. One considers that both the mechanical and electrical parts oscillate with the frequency of the external input voltage. The expressions of the electrical current and the mechanical displacement can be written as

$$
\begin{aligned}
& x=A \cos \left(\omega \tau+\phi_{1}\right) \\
& y=B \cos \left(\omega \tau+\phi_{2}\right) .
\end{aligned}
$$

$\phi_{1}$ and $\phi_{2}$ are the initial phases of the dynamical variables $x$ and $y$ while $A$ and $B$ are their respective amplitudes. The introduction of (7) into (6) leads to the following algebraic equations for $A$ and $B$ :

$$
\begin{aligned}
& \gamma_{m}^{2} A^{2} \\
& =\frac{9}{16} \alpha_{2}^{2} B^{6}+\frac{3}{2} \alpha_{2}\left(\alpha_{1}-\omega^{2}\right) B^{4} \\
& \quad+\left[\left(\alpha_{1}-\omega^{2}\right)^{2}+\beta^{2} \omega^{2}\right] B^{2} \\
& \frac{9}{16} \alpha_{2}^{2}\left(\lambda^{2}+\omega^{2}\right) B^{6}+\frac{3}{2} \alpha_{2} F B^{4}+G B^{2}=E_{0}^{2} \gamma_{m}^{2}
\end{aligned}
$$

with

$$
\begin{aligned}
F= & \left(\lambda^{2}+\omega^{2}\right)\left(\alpha_{1}-\omega^{2}\right)+\omega^{2} \gamma_{m} \gamma_{e} \\
G= & \left(\lambda^{2}+\omega^{2}\right)\left[\left(\alpha_{1}-\omega^{2}\right)^{2}+\beta^{2} \omega^{2}\right]+\omega^{2} \gamma_{m}^{2} \gamma_{e}^{2} \\
& +2 \omega^{2} \gamma_{m} \gamma_{e}\left(\alpha_{1}-\omega^{2}+\lambda \beta\right) .
\end{aligned}
$$

Figures 2(a) and 2(c) show that both the responses of the mechanical part and electrical part oscillate with perfect 


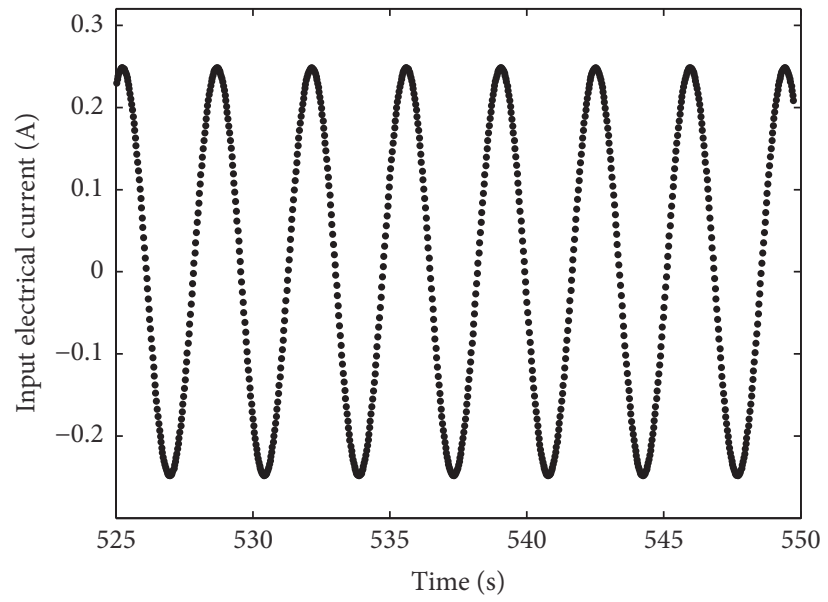

(a)

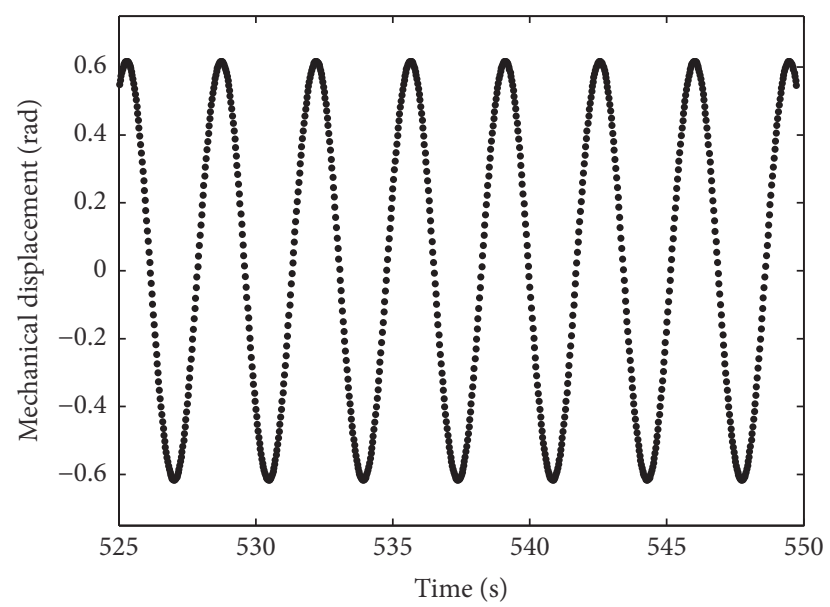

(c)

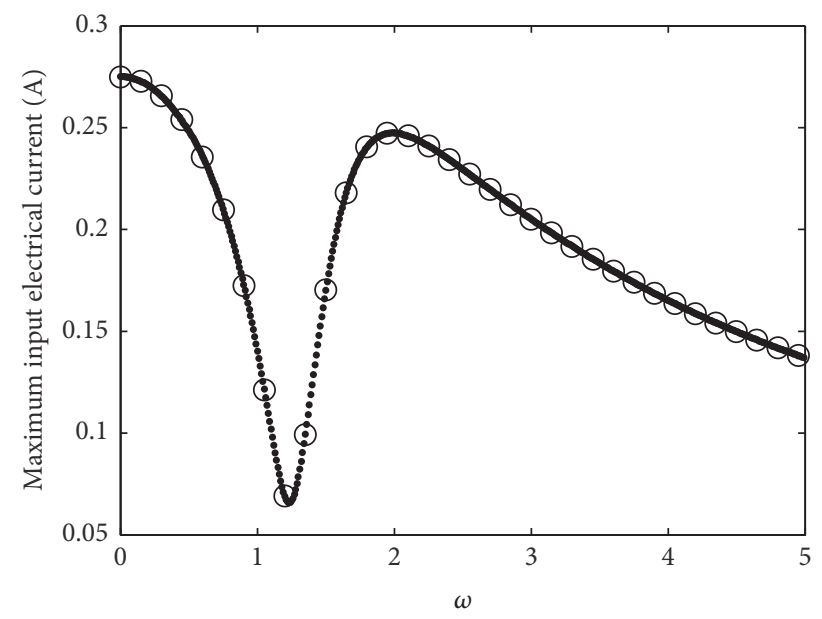

(b)

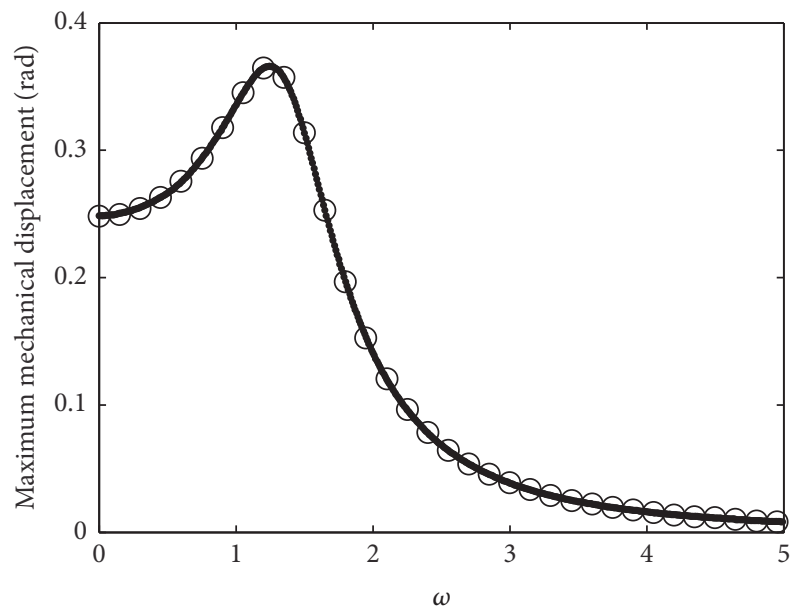

(d)

FIGURE 2: Times histories (graphs (a) and (c)) and the maximum amplitudes of the current and angular displacement versus the input frequency in unit of $w_{0}$ (graphs (b) and (d)) with the parameters of Table 1 and for $E_{0}=0.733(\omega=0.5$ for graphs (a) and (c)). The full black line represents the results from numerical simulation whereas the dot line is for the analytical derivation.

sinusoidal shape around the equilibrium position. This is due to the linear electromechanical coupling term that causes the mechanical and the electrical response to have the same dynamics. Moreover, the amplitudes of the dynamical variables depend on the frequency of the input signal as it appears in Figures 2(b) and 2(d). Indeed, an interesting fact appears as the frequency varies. One notes that the mechanical arm amplitude at resonance (the highest amplitude) is attained at a frequency where the electrical current is almost equal to zero. This corresponds to an optimal and recommended frequency because the consumption in terms of electrical energy is very low when the mechanical action is at its maximum level.

In order to have an extended view on the dynamical states of the robot arm, a bifurcation diagram is plotted in Figure 3 taking the excitation amplitude as the control parameter. The corresponding Lyapunov exponent is evaluated by generating the perturbation to the solutions of the dynamical equations (see (6)). If $\Delta x, \Delta y$, and $\Delta z$ are the perturbations of the dimensionless electrical current, angular displacement, and angular velocity, respectively, the perturbed solutions are $x^{\prime}=$ $x+\Delta x, y^{\prime}=y+\Delta y$, and $z^{\prime}=z+\Delta z[25]$. When they are introduced into (6) and separated (the perturbation from the dynamical variables), one obtains the following equations:

$$
\begin{aligned}
\dot{x}+\lambda x+\gamma_{e} \dot{y} & =E_{0} \cos (\omega \tau) \\
\Delta \dot{x}+\lambda \Delta x+\gamma_{e} \Delta \dot{y} & =0 \\
\ddot{y}+\beta \dot{y}+\sin \left(\theta_{0} y\right) & =\gamma_{m} x \\
\Delta \ddot{y}+\beta \Delta \dot{y}+\theta_{0} \Delta y \cos \left(\theta_{0} y\right) & =\gamma_{m} \Delta x .
\end{aligned}
$$

Then, the Lyapunov exponent can be calculated as [25]

$$
\text { Lya }=\lim _{t \rightarrow \infty} \frac{1}{t} \ln \sqrt{\Delta x^{2}+\Delta y^{2}+\Delta z^{2}} .
$$

As it appears in Figure 3, whatever the value of the parameter $E_{0}$, the system oscillates with a period equal to $m T$ where $T$ is 


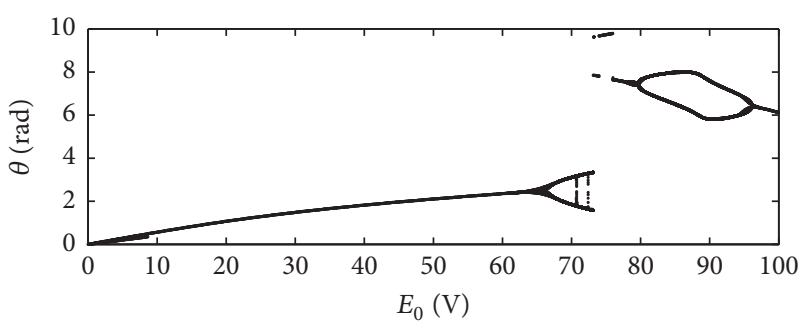

(a)

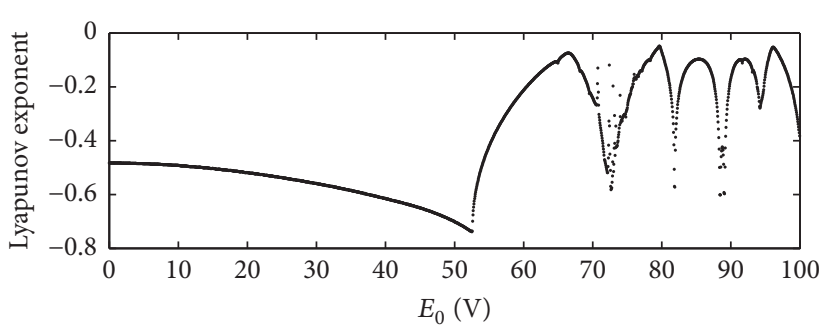

(b)

Figure 3: Bifurcation diagram (a) and Lyapunov exponent (b) of the mechanical arm as a function of the excitation amplitude. These graphs are plotted with the parameter values of Figure 2.

the period of the electrical source and $m$ is an integer. Indeed, the system alternates between the period-1T and period-2T oscillation from the origin $E_{0}$ close to 0 to $E_{0}=13$ (see Figure 3(a)). With the parameters values of Table 1 , a chaotic state is not observed as the Lyapunov exponent is always negative (Figure 3(b)). Some of the results obtained here are comparable to those of [11] where the authors found chaos and used the state-dependent Ricatti equation control and the nonlinear saturation control techniques to suppress the chaotic motion. These results are also comparable to those of [12] where the authors studied the rotation of a pendulum powered by an electromechanical excitation and observed period-1T rotation, period-1 $T$ oscillation, and period-2 $T$ oscillation.

\section{Effect of the Periodic Actions of the Load}

In this section, the effect of the periodic actions of the load mass is considered. This load $m_{0}$ is assumed to collide with the pendulum arm at the equilibrium position with the same frequency as the excitation frequency. The interaction results in impulsive forces or moments that have to be taken into consideration in the dynamical equations. The collision happens when the following conditions are satisfied:

$$
\begin{aligned}
& \theta=0, \\
& \frac{\mathrm{d} \theta}{\mathrm{d} t}>0 .
\end{aligned}
$$

The equation of the pendulum arm thus takes the following form:

$$
\frac{d^{2} \theta}{d \tau^{2}}+\omega_{1}^{2} \sin \theta+\mu \frac{d \theta}{d \tau}=\frac{n B b^{2}}{2} i-A \sum_{i=0}^{\infty} \delta(t-i T),
$$

where $A=\eta m_{0} g \ell$ is the amplitude of the perturbation, $\eta$ is the solid friction coefficient, $T=2 \pi / \Omega$, and $\delta$ is the Kronecker symbol. Using the rescalings $\xi=A / J \omega_{0}^{2} \theta_{0}$ and $\Gamma=\omega_{0} T$, the dimensionless equations are

$$
\begin{gathered}
\dot{x}+\lambda x+\gamma_{e} \dot{y}=E_{0} \cos (\omega \tau) \\
\ddot{y}+\beta \dot{y}+\sin \left(\theta_{0} y\right)=\gamma_{m} x-\xi \sum_{i=0}^{\infty} \delta(\tau-i \Gamma) .
\end{gathered}
$$

Figure 4 presents the time histories of the electrical and mechanical responses with different values of the collision amplitude. According to Figure 4(c), the curve representing the mechanical part reaches the positive size of the $y$-axis during oscillation. Consequently, the pendulum is able to move or to change the direction of the external mass $m_{0}$. But this action changes the shape of the signals compared to the case where $\xi=0$ (the case studied in Section 2): the angular displacement leaves the period-1T oscillation to period-2T oscillations while the electrical response changes from perfect sinusoidal oscillatory state to oscillation with fluctuations (compare Figure 4 and Figure 2 and c). However, in Figure 4(d), the amplitude $\xi$ of the perturbation is too large so that the pendulum moves only at the one side.

Figures 5(a) and 5(c) present the maximum amplitude of the electrical and mechanical parts of the system with impulsive force as a function of excitation frequency. Taking as reference the case without the impulsive force (case studied in Section 2), these Figures also show that, for low frequencies, the amplitude of the system with the load coincides with the case without load. But when the value of the frequency is large, the amplitude of the system with impulse force is larger than that of the system without the load. Moreover, for the range of large values of the excitation frequency, the collision can be perceived as a source of additional nonlinearity and leads to the jump phenomenon in the electrical and mechanical responses. In Figures 5(b) and 5(d), the amplitude curves are plotted as a function of the amplitude $\xi$ of the impulsive force. The jump phenomenon is also observed at a value of $\xi$ from which the pendulum is not able to move the load. The fluctuations in those two curves can be explained by the shape of Figures 4(b) and 4(d).

The bifurcation diagram plotted in Figure 6 shows periodic oscillations whatever the value of the parameter $\xi$. But this parameter causes the system to move from the period$1 T$ oscillations to period-2T oscillations and then period-3T oscillations. For large values of $\xi$, the dynamics of the system also change to small oscillations. Thus the simple sinusoidal responses (period- $T$ ) of Figure 2 are transformed into period$2 T$ and period-3T oscillations when the value of the mass to be pushed increases. 


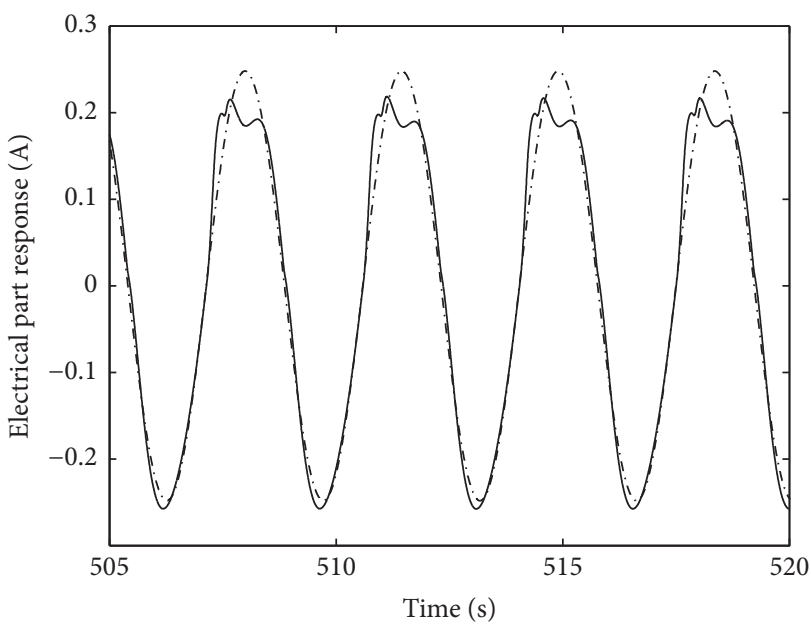

(a)

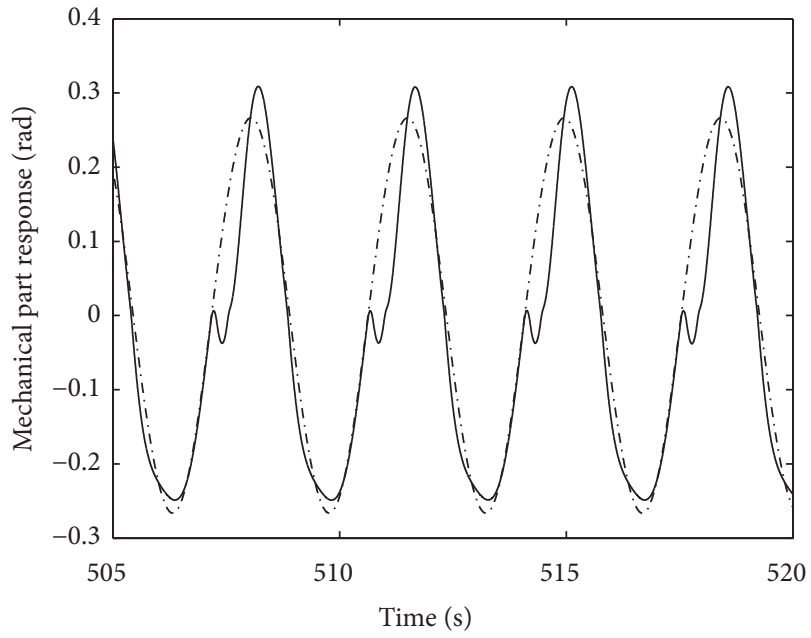

(c)

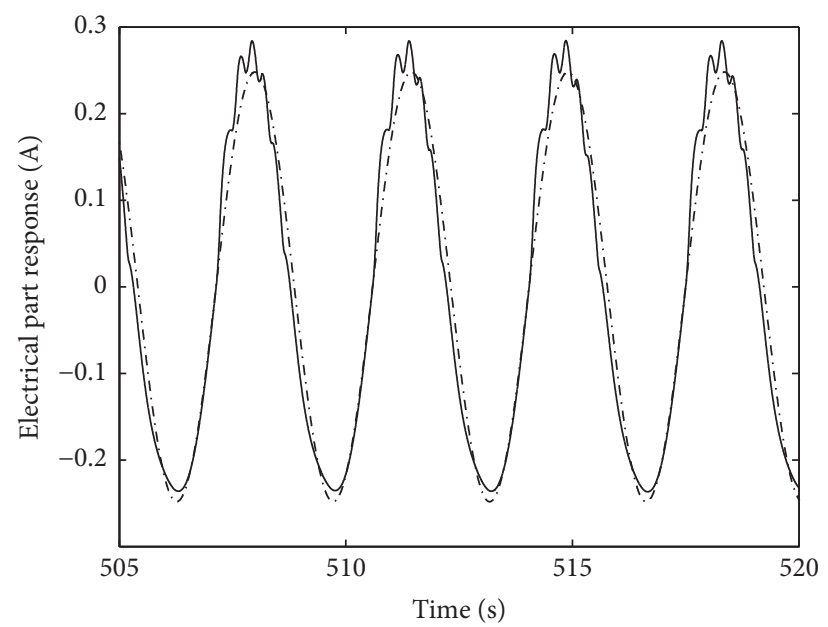

(b)

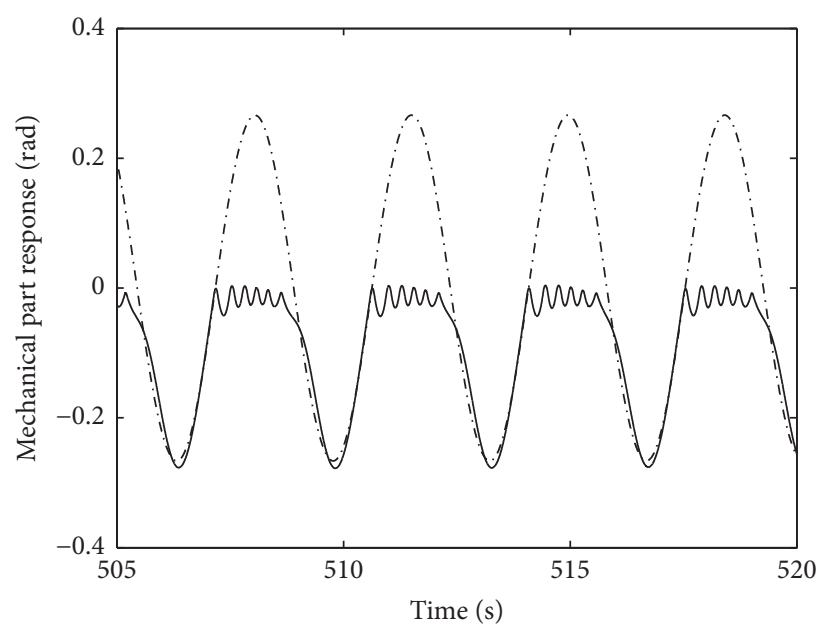

(d)

Figure 4: Electrical and mechanical response with respect to time in the presence (full line) and in the absence (dashed dotted line) of the impulsive contact force. With the parameters of Figure 2 and $\xi=0.3947$ (graphs (a) and (c)), $\xi=0.5921$ (graphs (b) and (d)).

\section{Control with Pulse-Like Voltage}

In order to reduce the level of distortion due to the collisions, one can add a periodic pulse-like electrical voltage to the sinusoidal one in the electrical part. The period of this new voltage should be equal to that of the collisions. Practically, this can be achieved by using a (piezoelectric or optical) detector/sensor which senses the arrivals of the loads of mass $m_{0}$ and commands, through a microcontroller, for instance, the generation of the pulse-like voltage. Under these conditions, the equations of the system become

$$
\begin{aligned}
& L \frac{d i}{d t}+R i+\frac{n B b^{2}}{2} \frac{d \theta}{d t}=e_{0} \cos (\Omega t)+e_{p} \sum_{i=0}^{\infty} \delta(t-i T) \\
& \frac{d^{2} \theta}{d t^{2}}+\omega_{1}^{2} \sin \theta+\mu \frac{d \theta}{d t}=\frac{n B b^{2}}{2} i-A \sum_{i=0}^{\infty} \delta(t-i T) .
\end{aligned}
$$

$e_{p}$ is the amplitude of the electrical pulse-like voltage. In the absence of the periodic signal, one just has to set $e_{0}=0$. The dimensionless forms of equations (16) are

$$
\begin{aligned}
\dot{x}+\lambda x+\gamma_{e} \dot{y} & =E_{0} \cos (\omega \tau)+E_{p} \sum_{i=0}^{\infty} \delta(\tau-i \Gamma) \\
\ddot{y}+\beta \dot{y}+\sin \left(\theta_{0} y\right) & =\gamma_{m} x-\xi \sum_{i=0}^{\infty} \delta(\tau-i \Gamma)
\end{aligned}
$$

with $E_{p}=e_{p} / J \omega_{0}^{2} \theta_{0}$ and other coefficients are defined in (2) and (14).

Two situations arise here. The first one corresponds to a configuration where the sinusoidal voltage is absent while, in the second situation, there is a mutual action of the sinusoidal and pulse-like voltages. The question to solve is to find the value of the amplitude EP of the pulse-like voltage which is able to push the mass $m_{0}$. Indeed, as noted in Figure 4, the pendulum arm is not able to push the mass but returns back after the collision. 


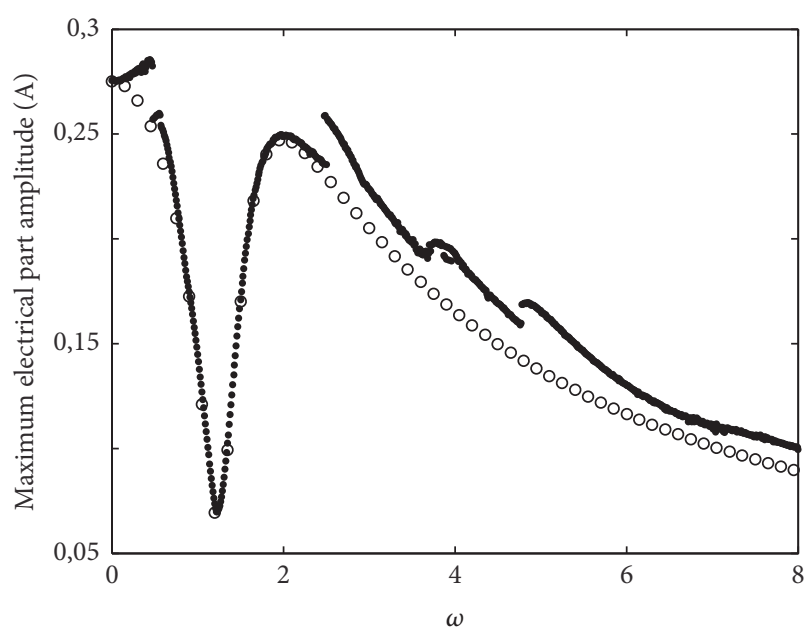

(a)

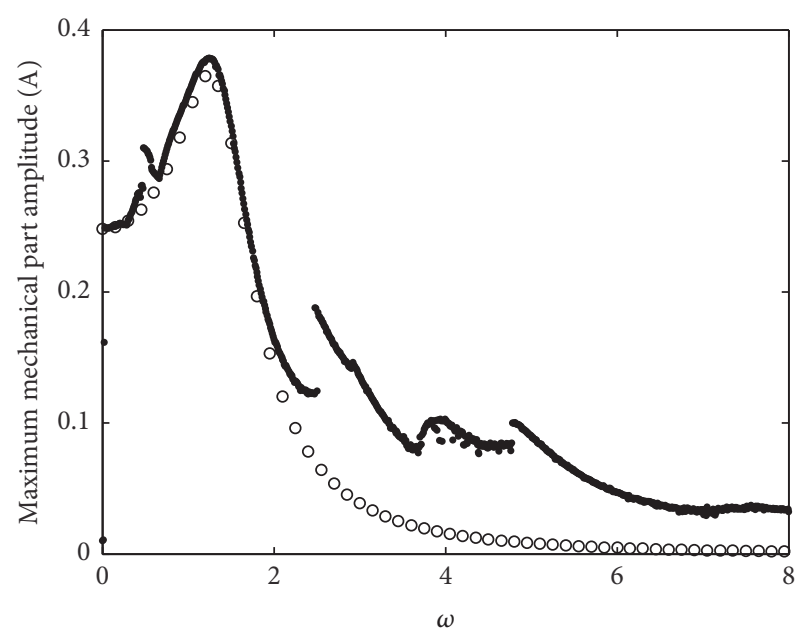

(c)

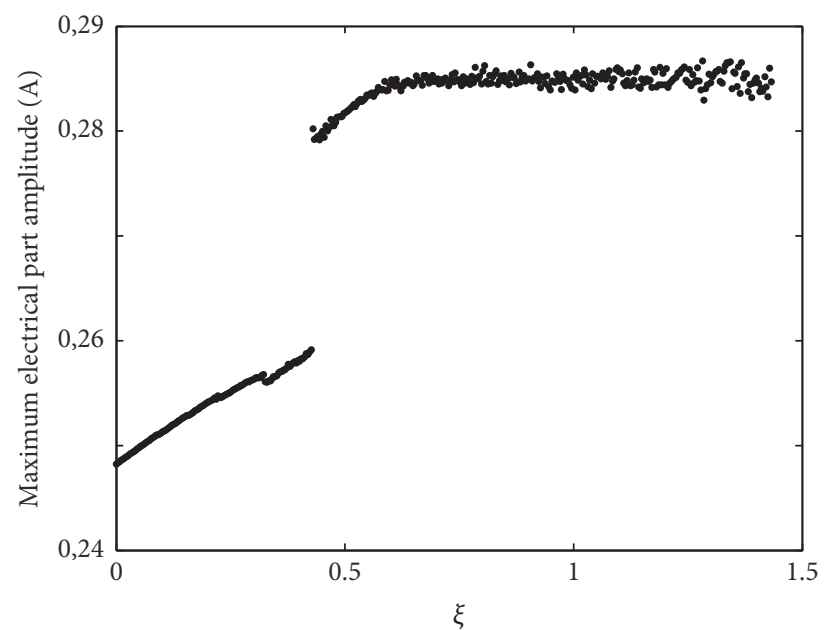

(b)

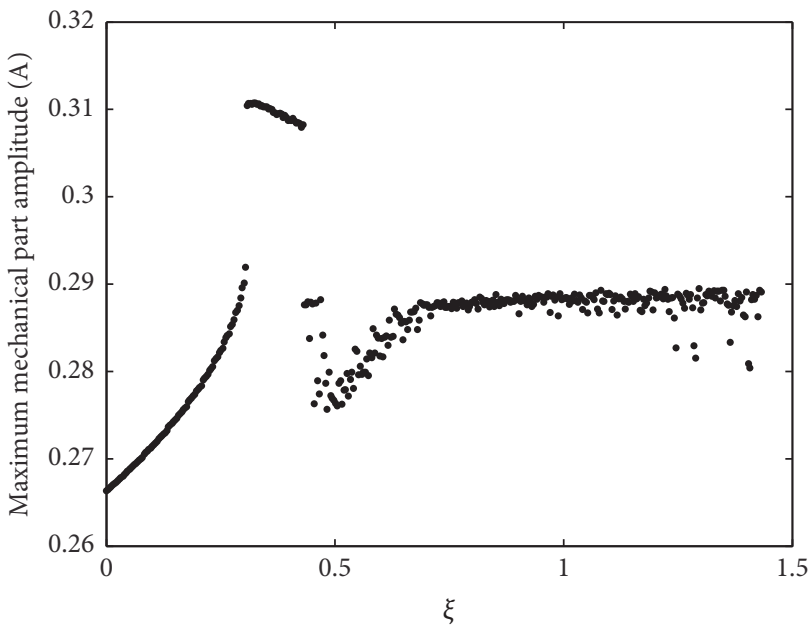

(d)

Figure 5: Maximum amplitude of the electrical and mechanical part with respect to the excitation frequency $\omega$ and the amplitude of the impulsive force $\xi$. In (a) and (c), the dot line represents the free pendulum case (case studied in Section 2 and Figure 2) and the solid line represents the case with the impulsive force. $\xi=0.3947$ for (a) and (c), while $\omega=0.5$ for (b) and (d).

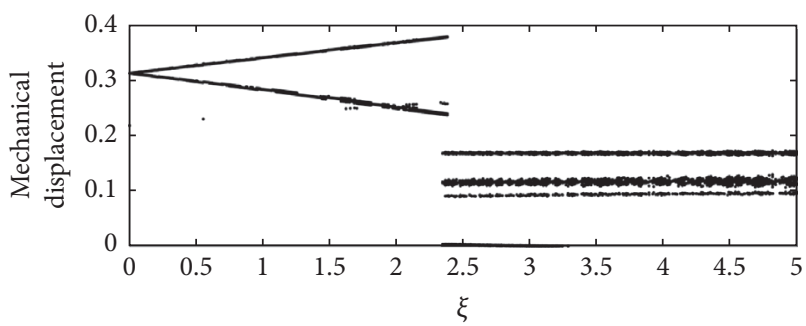

(a)

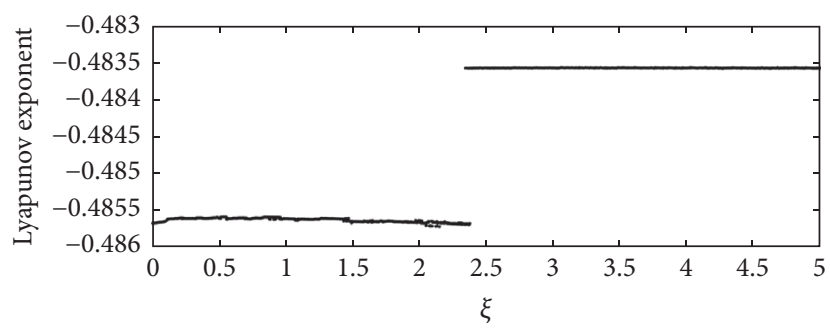

(b)

FIGURE 6: Bifurcation diagram (a) and Lyapunov exponent (b) of the mechanical arm with respect to the parameter $\xi$ considering the parameters of Figure 5(a).

Figure 7 presents in the $\left(E_{p}, m_{0} / m_{1}\right)$ plane the domain where the pushing action is effective. It corresponds to the shaded area of the figure. It is observed that when both signals are active, high masses can be pushed contrary to what is observed when only the pulse-like voltage is acting. However, the case where only the pulse-like signal is active is interesting since it corresponds to low consumption of energy because the pendulum arm is set into motion only when the mass is detected. The linear increase of the maximal value of $m_{0} / m_{1}$ versus Ep (in Figure 7(a)) may be related to the fact that Ep 


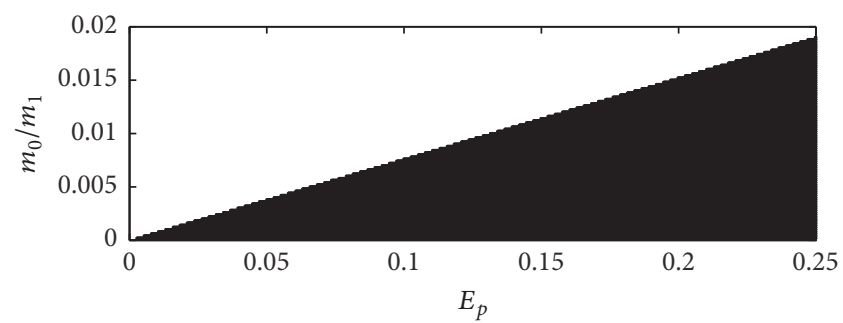

(a)

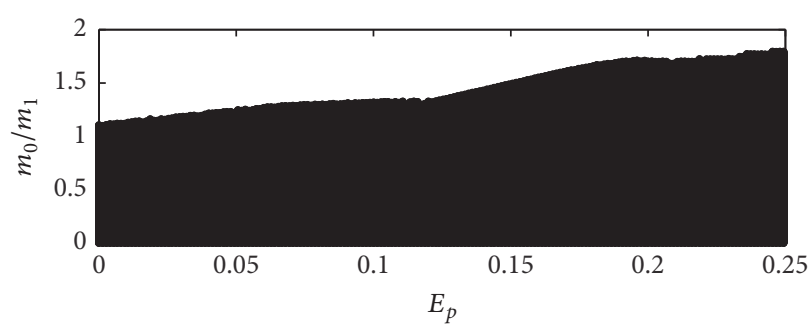

(b)

FIGURE 7: Regions of parameters leading to the motion (in black) of the external mass by the pendulum in the case of the single pulse excitation (a) and the sinusoidal excitation (b) in the $\left(E_{p}, m_{0} / m_{1}\right)$ space with $\xi=0.3947$ and $E_{0}=0.733$.

should directly compensate the factor $\xi$ proportional to the mass $m_{0}$ (see (18)).

\section{Efficiency of the Backstepping Control}

Another way to mitigate or reduce the distortion created by the collisions is to use an adaptive controller. The adaptive control is interesting as it can also mitigate the actions of other types of perturbations such as the change (continuous or stochastic) of the values of some parameters of the system. Indeed, during the functioning of the system, the environmental conditions, particularly the temperature, can force some intrinsic parameters of the system to change, for a short time or permanently, in an unknown way with regular or stochastic shape. For instance, the resistance and damping coefficients can change taking the values $\lambda^{\prime}=\lambda+\Delta \lambda$ and $\beta^{\prime}=\beta+\Delta \beta$, where $\Delta \lambda$ and $\Delta \beta$ are the bounded perturbations due to the electrical resistance and the dissipation variations.

The dynamics of the robot arm will also change consequently. In this situation, the adaptive controller is required since it adapts its own parameters to follow the unknown variations of the system parameters. Here, we develop a backstepping control strategy that will restore the system to its regular dynamics or considerably reduce the deviation from the normal working state.

To derive the backstepping controller, let us consider the reference system equation as

$$
\begin{aligned}
\dot{x}_{m}+\lambda x_{m}+\gamma_{e} \dot{y}_{m} & =E_{0} \cos (\omega t) \\
\ddot{y}_{m}+\beta \dot{y}_{m}+\alpha_{1} y_{m}+\alpha_{2} y_{m}^{3} & =\gamma_{m} x_{m} .
\end{aligned}
$$

Because of the perturbations, the actual state of the systems can become $x_{s}$ and $y_{s}$ satisfying (19a) and (19b), respectively. The perturbation can be of additive or multiplicative nature due, for instance, to the variations of the electric voltages or a short duration change in the system parameters. The dynamical state will deviate from its normal states $x_{m}$ and $y_{m}$. In order to force the system to return to its normal state, one should add a control function so that the equations are now

$$
\begin{aligned}
\dot{x}_{s}+\lambda x_{s}+\gamma_{e} \dot{y}_{s} & =E_{0} \cos (\omega \tau)-u \\
\ddot{y}_{s}+\beta \dot{y}_{s}+\alpha_{1} y_{s}+\alpha_{2} y_{s}^{3} & =\gamma_{m} x_{s} .
\end{aligned}
$$

Let us mention that since the perturbation is unknown, one assumes that the perturbed dynamical states $x_{s}$ and $y_{s}$ satisfy the differential equations of the system. The controller $u$ is appropriate if it leads $x_{s}$ and $y_{s}$ quickly to $x_{m}$ and $y_{m}$. The controller acts on the electrical circuit as in the case of the control of motors [26]. The controller returns to zero when the deviation is equal to zero. Let us mention that, for the sake of mathematical development, the Taylor expansion of the sine function is also used as when we were deriving the amplitude response curve in Section 2.

Denote

$$
\begin{aligned}
& e_{x}=x_{m}-x_{s} ; \\
& e_{y}=y_{m}-y_{s} ; \\
& e_{z}=z_{m}-z_{s} \\
& \varsigma=\alpha_{1}+3 \alpha_{2} y_{m}^{2},
\end{aligned}
$$

where $z_{m}=\dot{y}_{m}$ and $z_{s}=\dot{y}_{s}$.

The tracking error dynamics can be obtained in

$$
\begin{aligned}
& \dot{e}_{y}=e_{z} \\
& \dot{e}_{z}=\gamma_{m} e_{x}-\varsigma e_{y}-\beta e_{z} \\
& \dot{e}_{x}=u-\lambda e_{x}-\gamma_{e} e_{z} .
\end{aligned}
$$

Equations (22) have the strict feed-forward form. From (24), one can define a Lyapunov's function with some appropriate coefficients, conditions, and expression for the control law and then use some lemma, such as LaSalle Lemma [27], for the stability proof. A reverse way is used here where Lyapunov's function is constructed step after step and the resulting coefficients, conditions, and control law are defined and given. For this purpose, the adaptive backstepping method is used $[28,29]$. Let us consider the variable $z_{1}=e_{y}$. So

$$
\dot{z}_{1}=e_{z}=z_{2}+\mu_{1} .
$$

$\mu_{1}$ is the virtual controller. The Lyapunov function of this subsystem is given by

$$
V_{1}=\frac{1}{2} z_{1}^{2}
$$

Its derivative is expressed as

$$
\dot{V}_{1}=z_{1} \dot{z}_{1}=z_{1} z_{2}+z_{1} \mu_{1}
$$




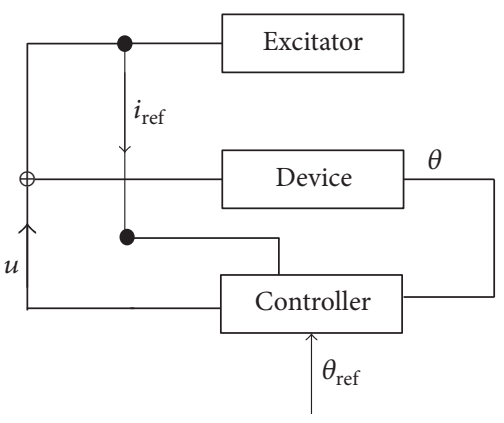

(a)

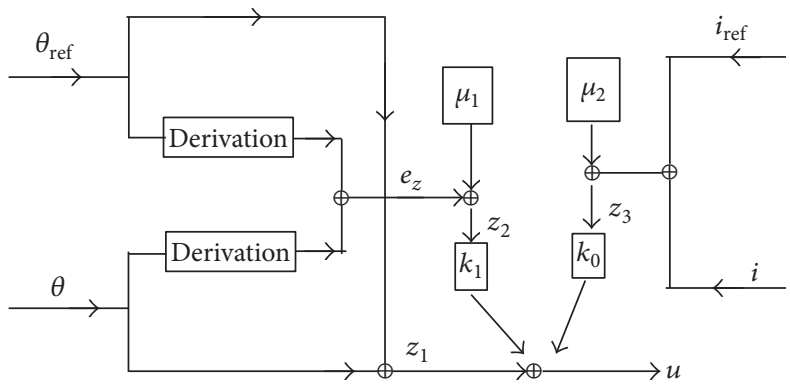

(b)

FIGURE 8: Block diagram of the adaptive backstepping control algorithm. $\oplus$ is the summation sign.

The controller $\mu_{1}$ can be taken as

$$
\mu_{1}=-h z_{1}
$$

where $h$ is a positive function defined as [29]

$$
\begin{aligned}
h(0) & =0 \\
\dot{h} & =\chi z_{1}^{2} .
\end{aligned}
$$

This definition of the function $h$ gives the controller the ability to adapt quickly and robustness depends of the value of $\chi$. Equation (25) becomes

$$
\dot{V}_{1}=-h z_{1}^{2}+z_{1} z_{2} .
$$

Then, the Lyapunov function of the second subsystem is

$$
V_{2}=V_{1}+\frac{1}{2} z_{2}^{2}
$$

Its derivative can be obtained as

$$
\begin{aligned}
\dot{V}_{2} & =-h z_{1}^{2}+z_{1} z_{2}+z_{2}\left[\gamma_{m} z_{3}-(\beta-h) z_{2}\right. \\
& \left.-\left(\zeta-\beta h+h^{2}\right) z_{1}+\gamma_{m} \mu_{2}\right]=-h z_{1}^{2}-(\beta-h) z_{2}^{2} \\
& +z_{2}\left[-\left(-1+\zeta-\beta h+h^{2}\right) z_{1}+\gamma_{m} \mu_{2}\right]+\gamma_{m} z_{3} z_{2} \\
& =-h z_{1}^{2}-(\beta-h) z_{2}^{2}+\gamma_{m} z_{3} z_{2}
\end{aligned}
$$

with $z_{3}=e_{x}-\mu_{2}$, and one takes take $\beta>h \forall t$ for negative coefficient of $z_{2}^{2}$ and

$$
\mu_{2}=\frac{-1+\zeta-\beta h+h^{2}}{\gamma_{m}} z_{1}=\sigma z_{1} .
$$

Finally, the Lyapunov function of the third subsystem of (22) is

$$
V_{3}=V_{2}+\frac{1}{2} z_{3}^{2}
$$

Its derivative can be given as

$$
\begin{aligned}
\dot{V}_{3} & \\
= & \dot{V}_{2} \\
& +z_{3}\left[u-\lambda z_{3}-\left(\gamma_{e}+\varsigma\right) z_{2}-\left(\lambda \sigma-\gamma_{e} h-h \sigma\right) z_{1}\right] \\
& =-h z_{1}^{2}-(\beta-h) z_{2}^{2}-k_{0} z_{3}^{2}
\end{aligned}
$$

with

$$
u=\left(\lambda \sigma-\gamma_{e} h-h \sigma\right) z_{1}+\left(\gamma_{e}+\varsigma-\gamma_{m}\right) z_{2}-k_{0} z_{3},
$$

where $k_{0}$ is the positive constant. Thus, through step-by-step adaptive backstepping method, the form of the controller is obtained at (34). Since $\beta>h, h>0$, and $k_{0}>0$, and with the expression of the control law (34), one finds that Lyapunov's function derivative is negative semidefinite. Equation (33) defines Lyapunov's function of the whole system in terms of variables $\left(z_{1}, z_{2}, z_{3}\right)$ coordinates. As written in the mathematical development above, the variables $\left(z_{1}, z_{2}, z_{3}\right)$ are expressed as $z_{1}=e_{y}, z_{2}=e_{z}-\mu_{1}$, and $z_{3}=e_{x}-\mu_{2}$. The fact that Lyapunov's function derivative is negative semidefinite ensures that the equilibrium state is locally asymptotically stable. This means that the small deviations will quickly go back to zero after the perturbation has ceased. As it was indicated before, another and usual way for the stability analysis is to first define a control law $u$ (e.g., (34)), with a function $h$ (see (27)) and a Lyapunov's function (e.g., (32)) and then show that Lyapunov's function derivative is negative semidefinite. But this strategy sometimes has some limit since it supposes an a priori known mathematical expression of the control law (which is difficult to define). A possible advantage of the procedure used here is that the control law appears from the mathematical procedure.

The controller of (34) can be connected to the device as shown in Figure 8. Figure 8(a) gives the complete block diagram and Figure 8(b) indicates how the controller is generated from the variables $z_{1}, z_{2}$, and $z_{3}$ which depend on the deviations measured by sensors.

The controller presented in (34) is applied on the pendulum dynamics when the effect of the load $m_{0}$ is absent or negligible (case of Section 2) and when it is taken into consideration (case of Section 3). Figures 9(a) and 9(b) present a perturbation resulting from a temporal variation of the parameters $\beta^{\prime}$ and $\lambda^{\prime}$. For a short period of time, the value of these parameters changes simultaneously. Figure 9(c) presents the error response of the mechanical part in the case where the control is absent and the case with control. One can note that the amplitude of the error when the control is applied is lower than in the case without control. This is the proof that the control is efficient at least in reducing the deviation from the normal state. Figure $9(\mathrm{~d})$ presents the 


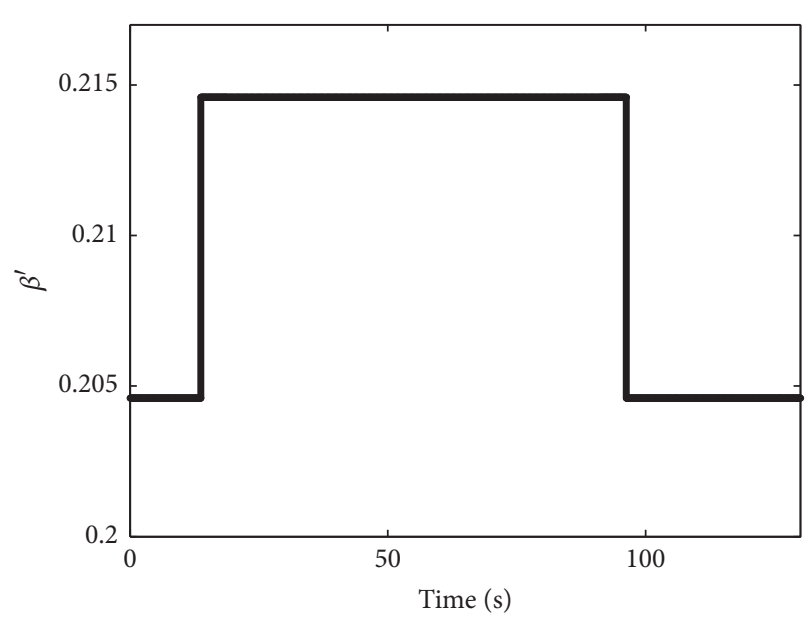

(a)

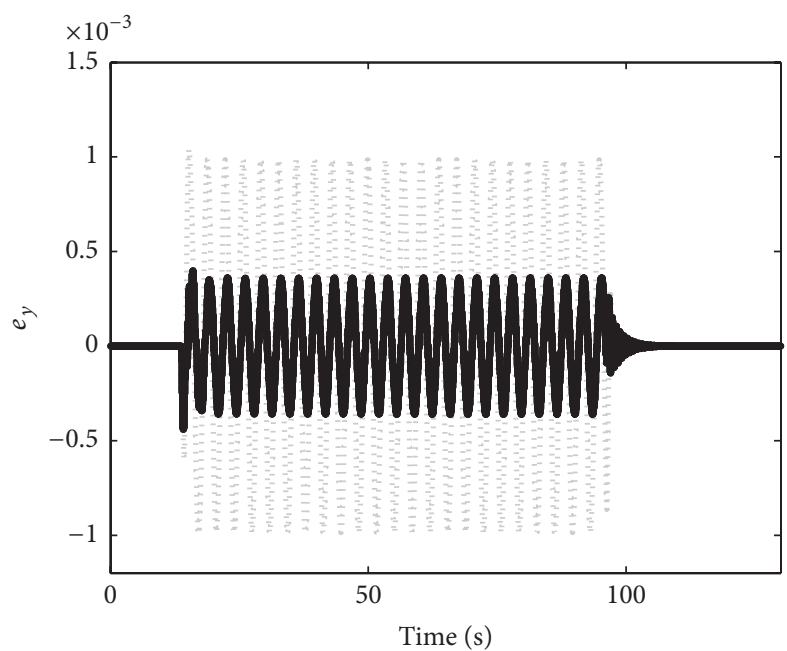

With control

Without control

(c)

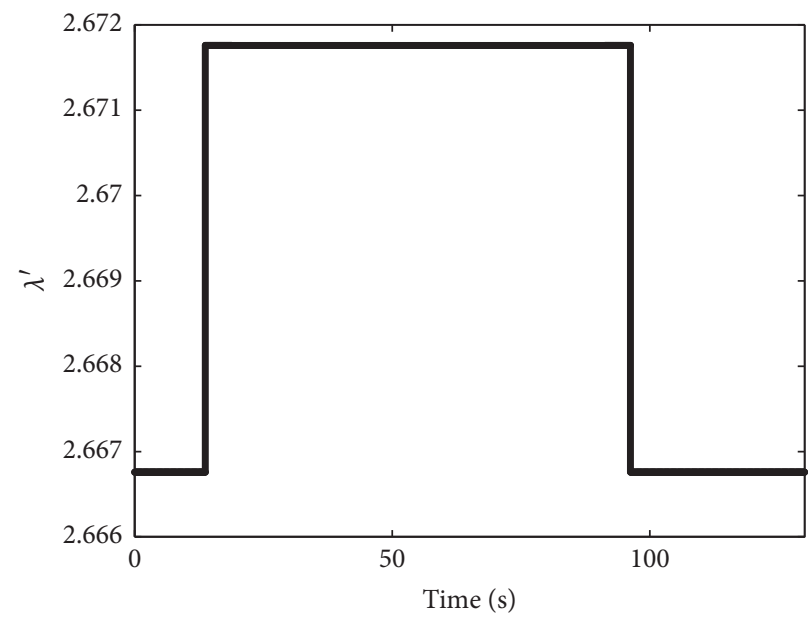

(b)

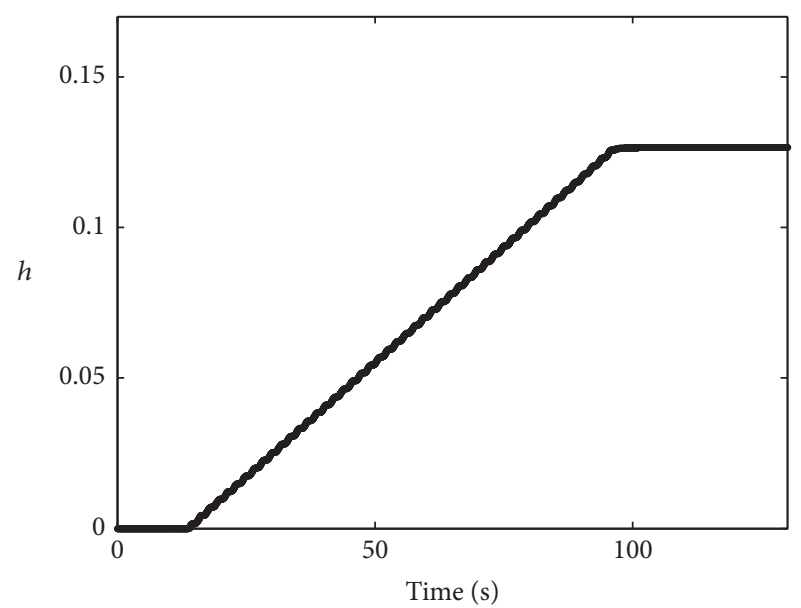

(d)

FIGURE 9: Times histories of the parameters $\beta^{\prime}(\mathrm{a})$ and $\lambda^{\prime}(\mathrm{b})$, the mechanical error $e_{y}(\mathrm{c})$, and the function $h(\mathrm{~d})$ in the case where the impulsive contact force is neglected $(\xi=0)$. Graph (c) represents in grey the mechanical error without the control and in black the mechanical error with the applied control with $k_{0}=2$ and $\chi=20$ and the parameters of Figure 5.

optimal value of the function $h$ rendering the adaptive control more efficient.

When the load $m_{0}$ is present in the absence of the variation of the resistance and damping coefficient, one obtains in Figure 10 that the controller tracks the perturbed trajectory into the normal one. This process results from the compensation by the backstepping controller of the distortions caused by the effect of the presence of the mass $m_{0}$ by the controller.

Considering now the case where the robot arm acts on the moving mass and also suffers the temporal variation of its parameters, Figure 11 shows that, even here, the backstepping controller is efficient since it considerably reduces the amplitude of the disturbance. The function $h$ increases differently compared to the case where the effect of the load $m_{0}$ is neglected.
An interesting question to raise is if the control strategy is efficient in a noisy environment where the parameters of the electromechanical device could vary randomly. This question has been considered here by assuming that the noisy environment influences the mechanical dissipation as expressed in

$$
\beta^{\prime \prime}=\beta+\psi(t)
$$

$\beta^{\prime \prime}$ is the mechanical dissipation perturbed by the noisy environment and $\psi(t)$ is the Gaussian white noise which is characterized by

$$
\begin{aligned}
\langle\psi(t)\rangle & =0 \\
\left\langle\psi(t), \psi\left(t^{\prime}\right)\right\rangle & =2 D \delta\left(t-t^{\prime}\right) .
\end{aligned}
$$




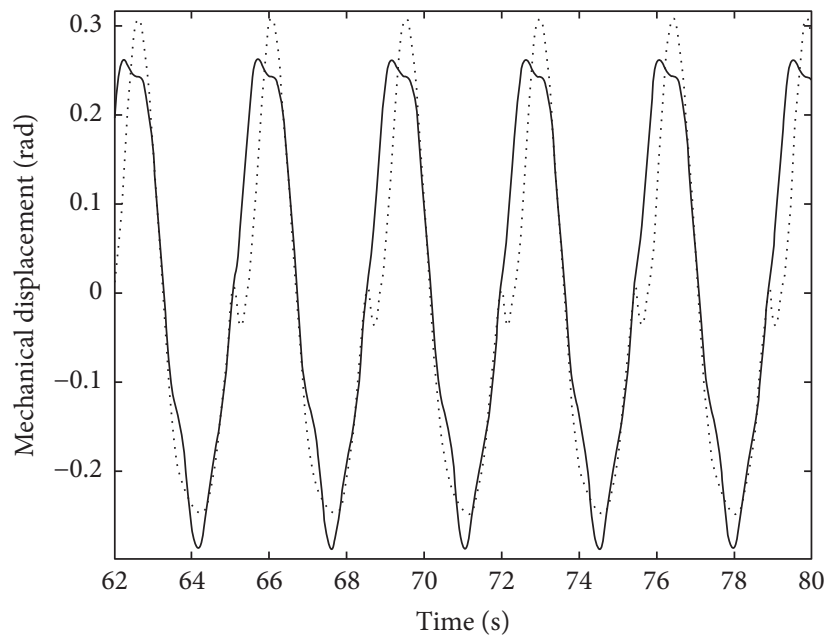

FIGURE 10: Times histories of the mechanical response with control (solid line) and without control (dotted line) in the case of the periodic impulsive contact force with the parameters of Figure $4(\mathrm{c})$ and $k_{0}=2$ and $\chi=0.005$.

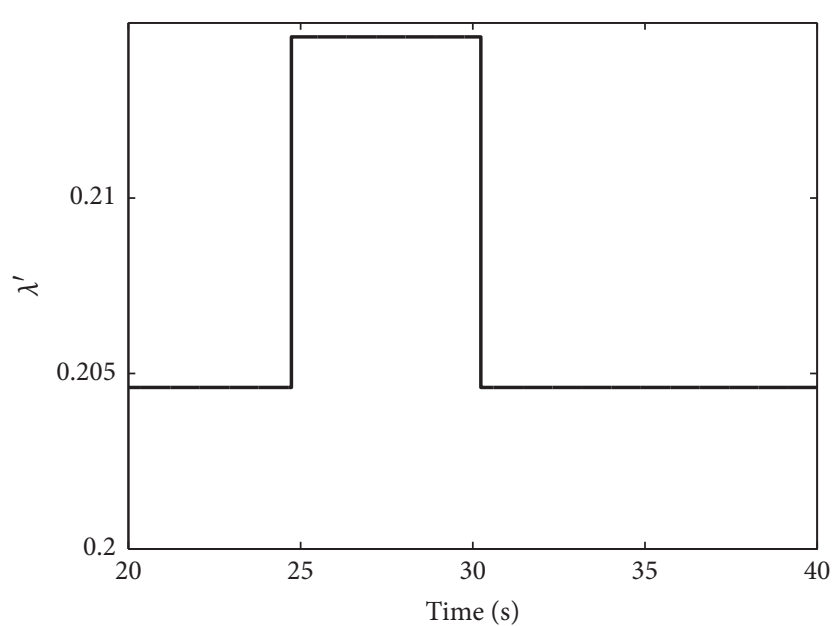

(a)

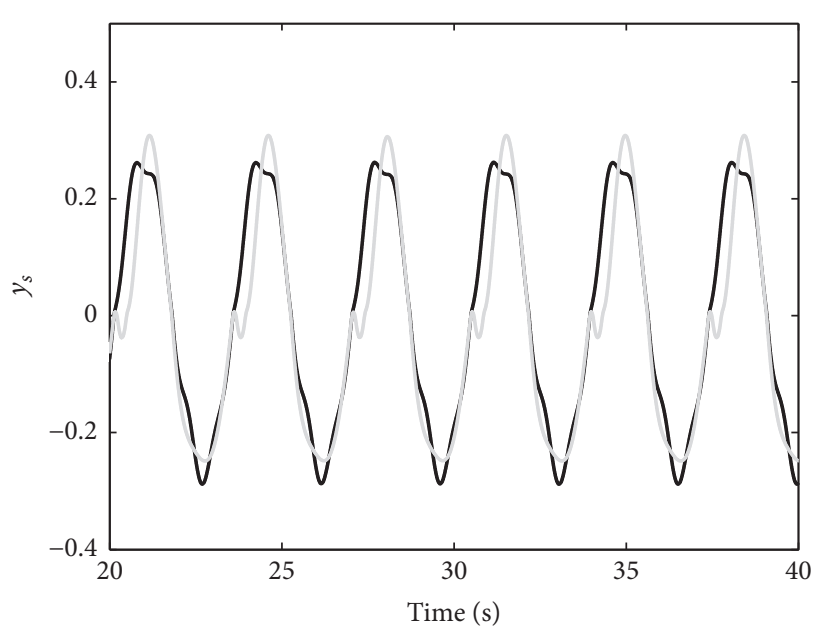

(c)

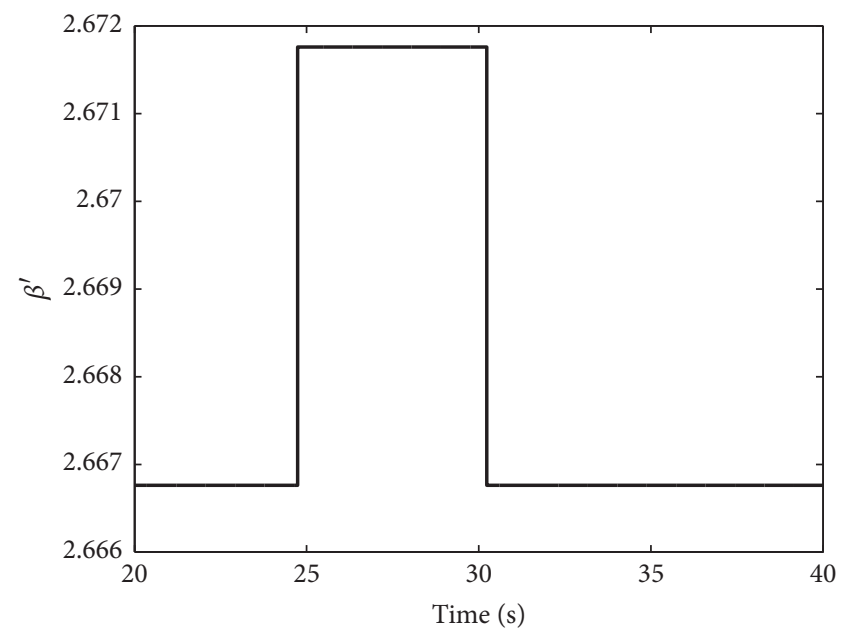

(b)

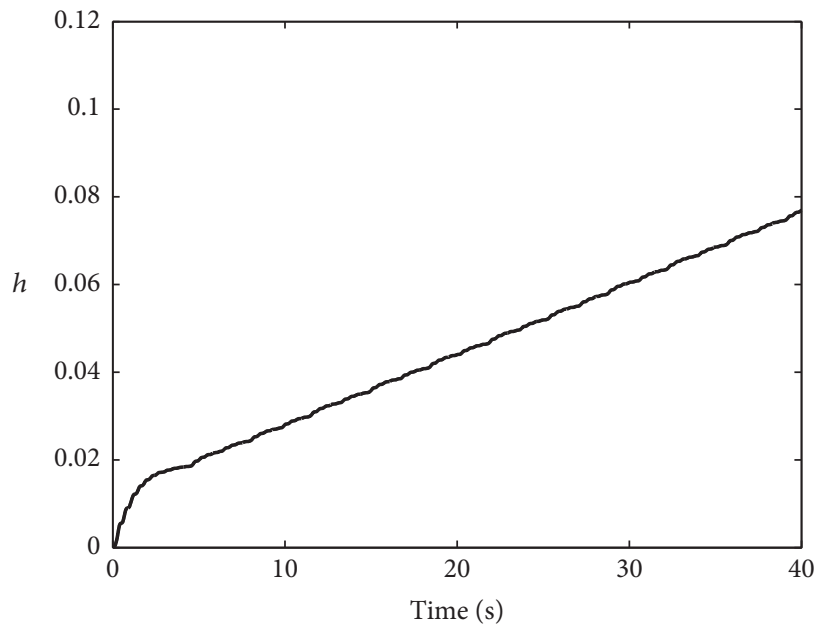

(d)

FIGURE 11: Times histories of the parameters $\beta^{\prime}$ (a) and $\lambda^{\prime}$ (b), the mechanical displacement $y$ (without control in grey and with control in black solid line) (c), and the function $h(\mathrm{~d})$ considering the impulsive contact force $(\xi=0.3947)$ with the parameters of Figure 9 . 


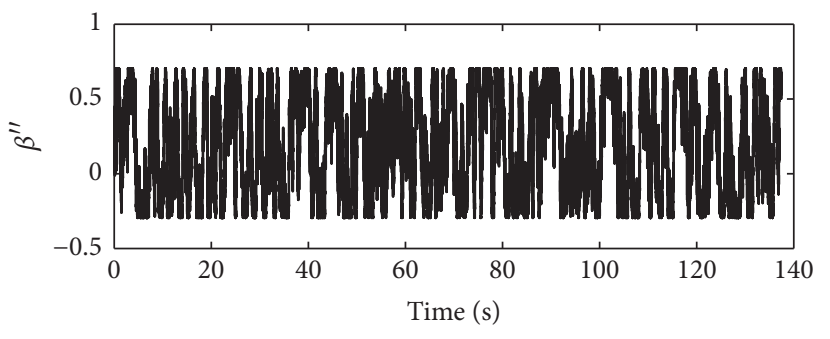

(a)

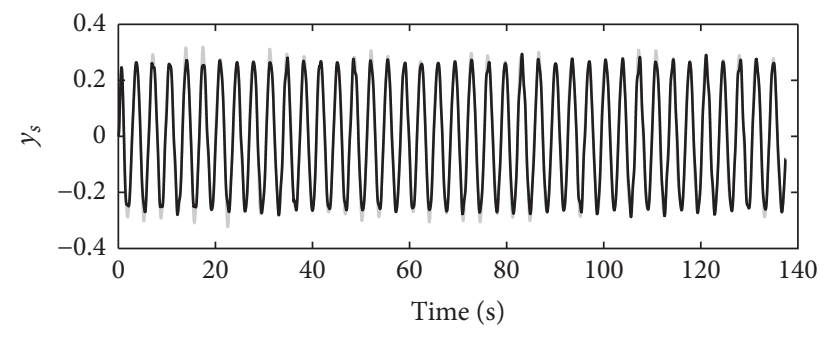

(b)

FIGURE 12: Time histories of the parameter $\beta^{\prime \prime}$ (a) and mechanical displacement (without control in grey and with control in solid black line) with $\xi=0$ and the parameters of Figure 9.

The use of additive Gaussian white noise is justified by the environmental origin of the noise and the fact that its modelling tends to the real conditions. In (20a) and (20b), $\beta^{\prime}$ is substituted by $\beta^{\prime \prime}$ and the new equations (stochastic differential equations) are solved numerically with the Kasdin algorithm, more appropriate for stochastic differential equations than the Runge-Kutta algorithm. The objective is to evaluate the performance of the controller obtained with the backstepping method. The strategy is to compare the controlled noisy dynamics to the reference dynamics of (19a) and (19b).

The results are presented in Figure 12. Figure 12(a) shows the fluctuations caused by the noisy environment conditions on the mechanical dissipation and Figure 12(b) shows how the controller reduces the deviation due to noises. This proves that the controller can operate in the noisy conditions to reduce the effects of perturbations and lead the dynamical state quickly to the almost normal state.

\section{Conclusion}

This work has dealt with the dynamics and control of an electromechanical robot arm manipulator pushing periodically a load. First neglecting the effect of the interaction with the load, the dynamics of the robot arm activated by a sinusoidal excitation has been characterized showing period$\mathrm{n} \mathrm{T}$ oscillations and delivering a mechanical resonance at very small electric current. The presence of the impulse force due to collisions has been seen to generate several behaviors such as jump of amplitude and distortions of the mechanical vibration and electrical current. An impulsive control has appeared to be able to mitigate the signal distortion. Added to that, an appropriate backstepping controller has been developed to reduce the amplitude of distortions due to the variations of some intrinsic parameters of the robot system and to the impulsive excitation resulting from the robot arm pushing actions or collisions.

\section{Conflicts of Interest}

The authors declare that they have no conflicts of interest.

\section{References}

[1] N. A. Chaturvedi, T. Lee, M. Leok, and N. H. McClamroch, "Nonlinear dynamics of the 3D pendulum," Journal of Nonlinear Science, vol. 21, no. 1, pp. 3-32, 2011.
[2] R. M. Ghigliazza and P. Holmes, "On the dynamics of cranes, or spherical pendula with moving supports," International Journal of Non-Linear Mechanics, vol. 37, no. 7, pp. 1211-1221, 2002.

[3] K. E. Rifai, G. Haller, and A. K. Bajaj, "Global dynamics of an autoparametric spring-mass-pendulum system," Nonlinear Dynamics, vol. 49, no. 1-2, pp. 105-116, 2007.

[4] N. Olgac and M. E. Cavdaroglu, "Full-state feedback controller design with "delay scheduling" for cart-and-pendulum dynamics," Mechatronics, vol. 21, no. 1, pp. 38-47, 2011.

[5] J. B. Mogo and P. Woafo, "Dynamics of a nonlinear electromechanical device with a pendulum arm," Journal of Computational and Nonlinear Dynamics, vol. 2, no. 4, pp. 374-378, 2007.

[6] S. Mohamed Sah, C. C. McGehee, and B. P. Mann, "Dynamics of a horizontal pendulum driven by high-frequency rocking," Journal of Sound and Vibration, vol. 332, no. 24, pp. 6505-6518, 2013.

[7] S. Sartori Junior, J. M. Balthazar, and B. R. Pontes Junior, "Nonlinear dynamics of a tower orchard sprayer based on an inverted pendulum model," Biosystems Engineering, vol. 103, no. 4, pp. 417-426, 2009.

[8] A. Y. T. Leung and J. L. Kuang, "On the chaotic dynamics of a spherical pendulum with a harmonically vibrating suspension," Nonlinear Dynamics, vol. 43, no. 3, pp. 213-238, 2006.

[9] C. A. K. Kwuimy and P. Woafo, "Dynamics, chaos and synchronization of self-sustained electromechanical systems with clamped-free flexible arm," Nonlinear Dynamics, vol. 53, no. 3, pp. 201-213, 2008.

[10] J. B. Mogo and P. Woafo, "Dynamics of a cantilever arm actuated by a nonlinear electrical circuit," Nonlinear Dynamics, vol. 63, no. 4, pp. 807-818, 2011.

[11] A. M. Tusset, V. c. Piccirillo, A. M. Bueno et al., "Chaos control and sensitivity analysis of a double pendulum arm excited by an RLC circuit based nonlinear shaker," Journal of Vibration and Control, vol. 22, no. 17, pp. 3621-3637, 2016.

[12] S.-H. Teh, K.-H. Chan, K.-C. Woo, and H. Demrdash, "Rotating a pendulum with an electromechanical excitation," International Journal of Non-Linear Mechanics, vol. 70, pp. 73-83, 2015.

[13] H. B. Fotsin and P. Woafo, "Adaptive synchronization of a modified and uncertain chaotic van der Pol-Duffing oscillator based on parameter identification," Chaos, Solitons and Fractals, vol. 24, no. 5, pp. 1363-1371, 2005.

[14] E. W. Bai and K. E. Lonngren, "Synchronization of two Lorenz systems using active control," Chaos, Solitons \& Fractals, vol. 8, no. 1, pp. 51-58, 1997.

[15] X. Tan, J. Zhang, and Y. Yang, "Synchronizing chaotic systems using backstepping design," Chaos, Solitons and Fractals, vol. 16, no. 1, pp. 37-45, 2003. 
[16] H. Zhang, X.-K. Ma, Y. Yang, and C.-D. Xu, “Generalized synchronization of hyperchaos and chaos using active backstepping design," Chinese Physics, vol. 14, no. 1, pp. 86-94, 2005.

[17] I. Eker and Ş. A. Akınal, "Sliding mode control with integral augmented sliding surface: design and experimental application to an electromechanical system," Electrical Engineering, vol. 90, no. 3, pp. 189-197, 2008.

[18] A. Shiriaev, A. Pogromsky, H. Ludvigsen, and O. Egeland, "On global properties of passivity-based control of an inverted pendulum," International Journal of Robust and Nonlinear Control, vol. 10, no. 4, pp. 283-300, 2000.

[19] Q. Wei, W. P. Dayawansa, and W. S. Levine, "Nonlinear controller for an inverted pendulum having restricted travel," Automatica, vol. 31, no. 6, pp. 841-850, 1995.

[20] C. C. Chung and J. Hauser, "Nonlinear control of a swinging pendulum," Automatica, vol. 31, no. 6, pp. 851-862, 1995.

[21] S. Riachy, Y. Orlov, T. Floquet, R. Santiesteban, and J.-P. Richard, "Second-order sliding mode control of underactuated mechanical systems. I. Local stabilization with application to an inverted pendulum," International Journal of Robust and Nonlinear Control, vol. 18, no. 4-5, pp. 529-543, 2008.

[22] B. Zhao, M. Li, H. Yu, H. Hu, and L. Sun, "Dynamics and motion control of a two pendulums driven spherical robot," in Proceedings of the 23rd IEEE/RSJ 2010 International Conference on Intelligent Robots and Systems (IROS '10), pp. 147-153, Taipei, Taiwan, October 2010.

[23] A. S. De Paula, M. A. Savi, M. Wiercigroch, and E. Pavlovskaia, "Bifurcation control of a parametric pendulum," International Journal of Bifurcation and Chaos, vol. 22, no. 5, Article ID 1250111, 2012.

[24] A. N. Kadjie and P. Woafo, "Effects of springs on a pendulum electromechanical energy harvester," Theoretical \& Applied Mechanics Letters, vol. 4, no. 6, Article ID 063001, 2014.

[25] D. O. Tcheutchoua Fossi and P. Woafo, "Dynamics of an electromechanical system with angular and ferroresonant nonlinearities," Journal of Vibration and Acoustics, vol. 133, no. 6, Article ID 061018, 2011.

[26] B. Rashidi, M. Esmaeilpour, and M. R. Homaeinezhad, "Precise angular speed control of permanent magnet DC motors in presence of high modeling uncertainties via sliding mode observerbased model reference adaptive algorithm," Mechatronics, vol. 28, pp. 79-95, 2015.

[27] J. P. Lasalle, "Some Extensions of Liapunov's Second Method," IRE Transactions on Circuit Theory, vol. 7, no. 4, pp. 520-527, 1960.

[28] W. Zhen, W. Yuntian, L. Yongxin, and Z. Yongjie, "Adaptive backstepping control of a nonlinear electromechanical system with unknown parameters," in Proceedings of the 2009 4th International Conference on Computer Science and Education, ICCSE, pp. 441-444, IEEE, Nanning, China, July 2009.

[29] E. W. Chimi Kontchou, H. B. Fotsin, and P. Woafo, "Feedback control and adaptive synchronization of chaotic forced Bonhoeffer-van der Pol oscillators," Physica Scripta, vol. 77, no. 4, Article ID 045001, 2008. 


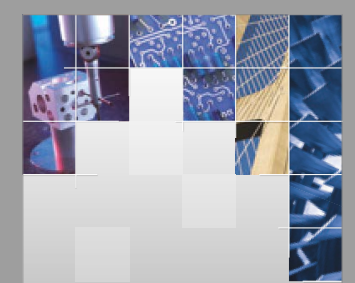

\section{Enfincering}
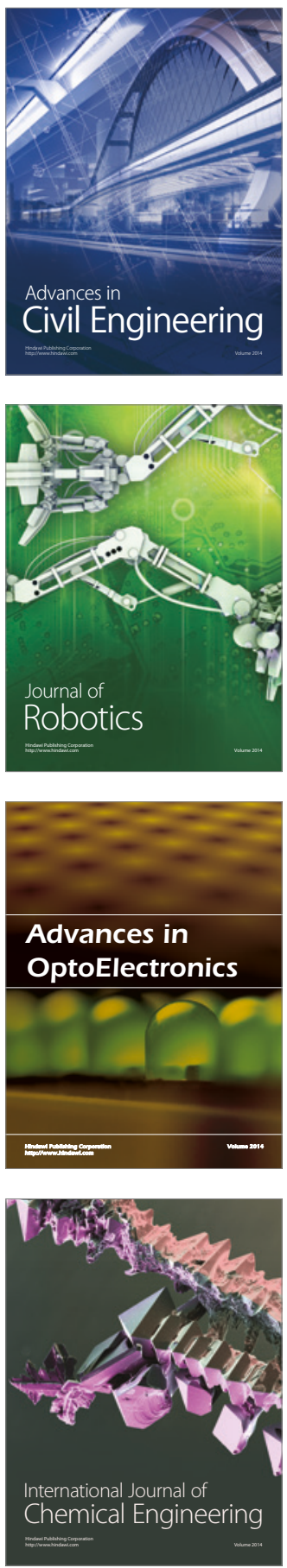

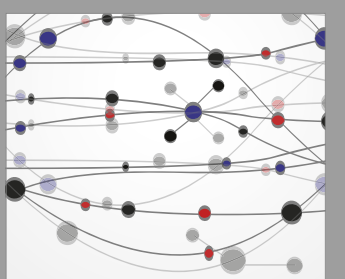

The Scientific World Journal

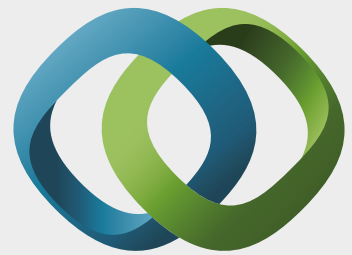

\section{Hindawi}

Submit your manuscripts at

https://www.hindawi.com
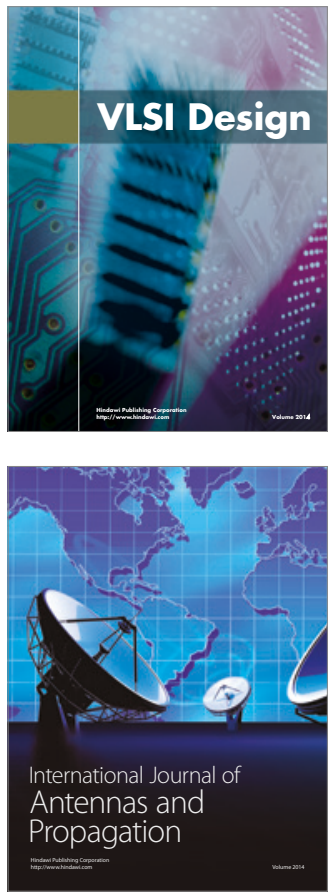

\section{Rotating}

Machinery
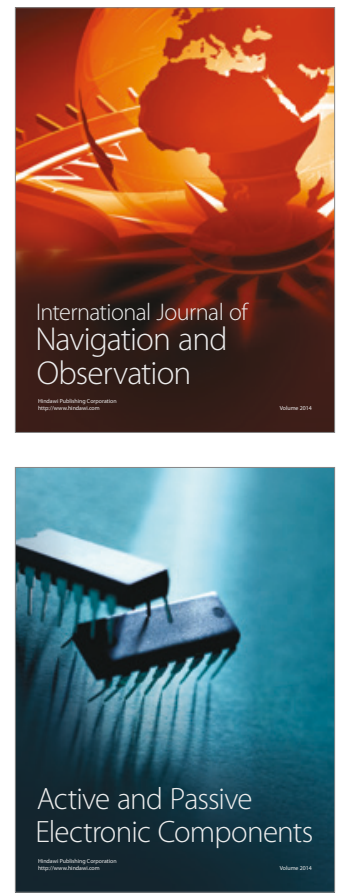
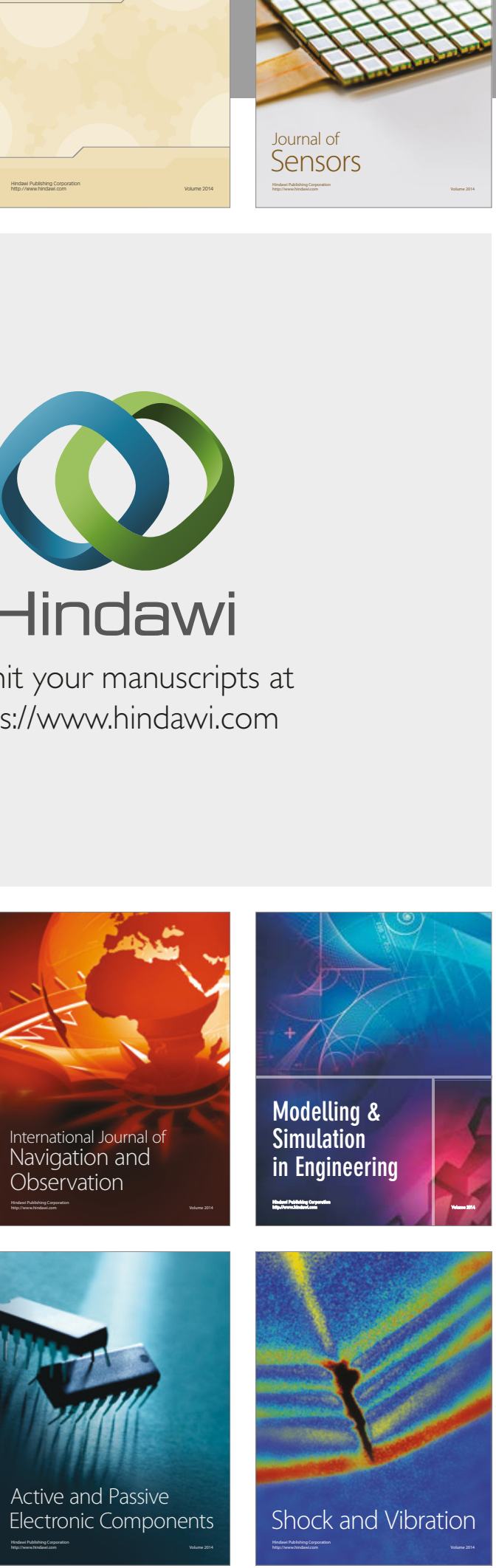
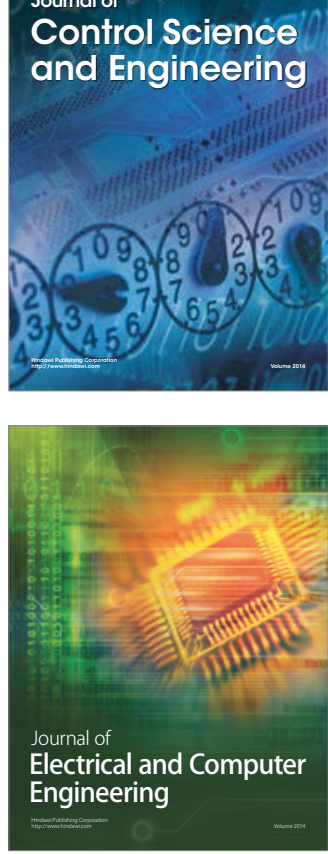

Distributed

Journal of

Control Science

and Engineering
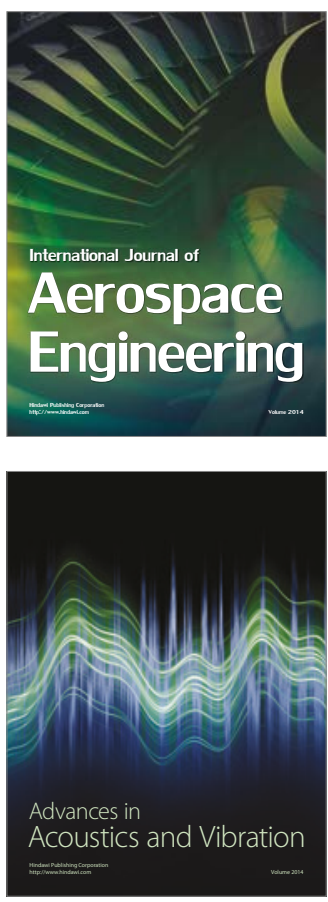

Sensor Networks 